\title{
Regarder sans voir : impact de conversations téléphoniques courtes sur l'attention visuelle en conduite simulée
}

\section{Looked but failed to see: Effect of short phone conversations on visual attention in simulated driving}

\author{
Céline Lemercier · Julien Tardieu - Pascal Gaillard P Pierre-Vincent Paubel \\ C IFSTTAR et Éditions NecPlus 2016
}

Résumé L’inattention au volant est définie comme la réorientation de l'attention du conducteur du traitement de la scène routière vers le traitement d'autres sources d'information (téléphoner, penser...). Inspirée par l'étude de Strayer et al. (2003, Expérience 3), l'étude présente investigue l'aveuglement inattentionnel à l'environnement routier provoqué par des conversations téléphoniques de courte durée $(<20 \mathrm{sec}$.) et factuelles. Le protocole consistait en un parcours autoroutier simulé alternant des périodes de conduite seule et de conduite en téléphonant. Sur le parcours, 30 panneaux sur portique présentant des noms communs étaient éparpillés tels que la moitié étaient vus en conduite seule et l'autre en conduite en téléphonant. Les communications téléphoniques consistaient en un échange de questions/réponses. Durant le parcours, le comportement oculométrique du conducteur était enregistré. Une épreuve de reconnaissance incidente des panneaux routiers était ensuite proposée. Les noms présentés en situation de conduite en téléphonant sont moins bien reconnus, sans que

Céline Lemercier $(\bowtie)$

Université de Toulouse, CLLE-Laboratoire Travail et Cognition, UMR CNRS 5263

5, allée Antonio Machado, 31058 Toulouse Cedex 01, France

e-mail : celine.lemercier@univ-tlse2.fr

Julien Tardieu( $\bowtie)$

Université de Toulouse, MSHS-T USR3414,

5, allée Antonio Machado, 31058 Toulouse Cedex 01, France

e-mail : julien.tardieu@univ-tlse2.fr

Pascal Gaillard( $\square)$

Université de Toulouse, CLLE-Laboratoire Travail et Cognition, UMR CNRS 5263

5, allée Antonio Machado, 31058 Toulouse Cedex 01, France

e-mail : pascal.gaillard@univ-tlse2.fr

Pierre-Vincent Paubel $(\triangle)$

Université de Toulouse, CLLE-Laboratoire Travail et Cognition, UMR CNRS 5263

5, allée Antonio Machado, 31058 Toulouse Cedex 01, France

e-mail : paubel@univ-tlse2.fr les paramètres oculaires ne diffèrent significativement de la situation de conduite seule. Comme lors de communications de plus longues durées, les conducteurs seraient aveugles inattentivement à la scène routière. Les implications de ces résultats sont discutées au regard d'autres sources d'inattention au volant.

Mots clés téléphone et conduite automobile simulée . attention · inattention · aveuglement inattentionnel · dilatation pupillaire $\cdot$ fixations oculaires

\begin{abstract}
The inattention in the steering wheel is defined as the reorientation of the driver's attention from the processing of the road scene to the processing of the other information sources (phoning, thinking...). Inspired by the Strayer and al.' study (2003, Experiment 3), the present study investigates the inattentionnal blindness to the road environment provoked by short-lasting $(<20 \mathrm{sec})$ and factual phone conversations. The protocol consisted of a motorway circuit alternating "only driving" and "driving and phoning" periods. On the motorway, 30 panels on portico presenting common nouns were scattered such as half were seen in "only driving" period and the other one in "driving and phoning" period. Phone conversations consisted of an exchange of questions/answers. During driving session, the ocular behavior drivers was recorded. An incidental recognition test of road panels was then proposed. Names presented in "driving and phoning" period are less correctly recognized, without the eye parameters differ significantly from the situation of "only driving" period. As during communications of more long-lasting, the drivers would be as inattentively blind to the road scene. The implications of these results are discussed with regard to other sources of inattention in driving.
\end{abstract}

Keywords Phone and simulated driving - attention . inattention - inattentional blindness - pupillary dilatation . ocular fixations. 


\section{Introduction}

Alors que vous êtes au volant de votre véhicule, bien concentré sur la scène routière, la radio passe le morceau de musique sur lequel vous avez dansé pour la première fois avec votre ami(e). Plus tard, alors que vous êtes à nouveau focalisé sur votre activité de conduite, votre collègue vous téléphone sur votre mobile et vous soumet un problème auquel il est confronté au sujet d'une panne matérielle quelconque. Plus tard encore, vous passez devant une affiche promotionnelle portant sur des bananes, vous vous souvenez alors que vous n'avez plus de pain et qu'il vous faut en acheter avant de rentrer chez vous. L'ensemble de ces situations fait référence à un même phénomène : l'inattention au volant [1].

Bien que peu étudiée expérimentalement, l'inattention est pourtant considérée comme l'un des facteurs déterminants dans les accidents de la route depuis la fin des années 1970 $[2,3]$. Dès 1979, Treat considère ainsi que l'inattention est, avec la surveillance inexacte de la route, la principale cause des accidents examinés. Similairement, Hendricks, Fell et Freedman [4] constatent que $22,7 \%$ des 723 accidents recensés dans leur étude sont imputables directement à une inattention du conducteur. Pour Rumar [5] l'inattention au volant expliquerait les erreurs de détection tardive des autres usagers de la route (piétons, motocyclistes), responsable des collisions avec ces derniers.

Alors que le conducteur est focalisé sur son activité principale de conduite, l'arrivée d'un événement particulier (conversation téléphonique, extrait musical indiçant un souvenir passé, lecture d'une affiche de magasin indiçant un souvenir du futur) va déclencher le traitement de pensées internes. Le point de focalisation de l'attention se déplace subséquemment du traitement des informations issues de la scène routière vers l'objet de ses pensées : Le conducteur est inattentif au volant, dans le sens où le contrôle qu'il exerçait sur son environnement devient secondaire, impliquant une dégradation de son comportement de conduite [1]. En accord avec les études antérieures, nous considérons ici que le sujet humain est inattentif lorsqu'il néglige le traitement des informations critiques à sa sécurité, la distraction étant un cas particulier d'inattention au volant [6]. Récemment, une étude menée par Pêcher et Lemercier [7] a ainsi révélé une dégradation du comportement attentionnel sélectif $\mathrm{du}$ conducteur suite à une induction émotionnelle de tristesse. Utilisant une version adaptée du protocole de Fan et al. [8) sur simulateur de conduite automobile, les auteurs ont montré une altération spécifique de la fonction d'orientation de l'attention chez le conducteur triste. Absorbé par des pensées ruminatives, le conducteur se comporte alors comme s'il était aveugle au contenu des informations spatiales délivrées par l'environnement routier [8). L'aveuglement, tel qu'il est abordé dans cette étude, ne renvoie pas à une défaillance sensorielle mais plutôt à une défaillance d'ordre perceptivo-attentionnelle, appelée cécité inattentionnelle [10] ou phénomène du « regarder sans voir » (Looked But Failed To See ; LBFTS) [11].

Découverte par Neisser, la cécité inattentionnelle réfère à l'incapacité à détecter dans une scène visuelle un objet à la fois saillant et inattendu lorsque l'attention visuelle est engagée par ailleurs, et ce, même si l'objet se trouve dans le focus visuel [12]. Elle serait la conséquence d'une orientation discrète (dans le sens où ce mouvement attentionnel n'est pas accompagné d'un mouvement visuel) de l'attention spatiale du sujet loin de l'item en question. En d'autres termes, l'engagement de l'attention sur le traitement sélectif d'une information particulière provoquerait une absence de traitement attentionnel explicite de l'objet saillant et inattendu [13]. À ce jour, l'étude de la cécité inattentionnelle au volant a fait l'objet d'un nombre restreint de publications $[14,15,16]$. Parmi elles, l'étude menée par Strayer, Drews et Johnston [15, Expérience 3] montre que l'engagement du conducteur dans une conversation téléphonique attrayante (dans le sens où elle a trait à un thème d'intérêt du conducteur, défini sur la base d'un questionnaire complété préalablement par le conducteur) entraîne une cécité inattentionnelle du conducteur à l'environnement routier. Les participants conduisaient sur un parcours urbain simulé intégrant 6 sections de $1,9 \mathrm{~km}$ chacune (dont la moitié en condition de conduite seule et l'autre moitié en condition de conduite en téléphonant), sur le parcours 30 panneaux publicitaires étaient présentés, 15 sur les sections de conduite seule et 15 sur les sections de conduite en téléphonant. Durant toute la durée du trajet, les mouvements oculaires des conducteurs étaient enregistrés. Juste après avoir effectué ce parcours, un test de reconnaissance incident des panneaux publicitaires était alors complété par les conducteurs. Les auteurs montrent une dégradation de la performance de reconnaissance explicite des panneaux publicitaires en condition de conduite avec conversation téléphonique, sans altération des paramètres oculaires analysés (durée des fixations oculaires, fréquences des fixations oculaires). Ils concluent que l'engagement du conducteur dans des conversations téléphoniques d'intérêt et d'une durée d'environ 2 minutes, entrainerait un déplacement de l'attention de la scène routière vers le sujet de la conversation. La conséquence en est une altération du traitement par l'attention des objets environnementaux. Notons que la durée des communications téléphoniques est ici estimée à partir des données issues du protocole de Strayer et al. [15] sur la vitesse prescrite de conduite (50 km/h en ville), et de longueur des sections intégrant la conversation $(1,9 \mathrm{~km})$.

D'après les résultats de l'enquête menée par Brusque et Alauzet en 2008 [18] sur les Français et le mobile au volant, la durée moyenne de conversation d'un conducteur 
est de 3 minutes. En pratique, nombre de communications téléphoniques au volant se résument à un échange rapide avec l'interlocuteur téléphonique et portent sur des préoccupations factuelles, de la vie courante (Qui va chercher les enfants à l'école ? Dans quelle salle se déroule la prochaine réunion ? etc.). L'impact de ce type de conversations à la fois de très courte durée $(<30$ secondes) et peu engageantes n'a, à notre connaissance, jusqu'à présent fait l'objet d'aucune étude en conduite. Sans doute, sont-elles considérées de moindre dangerosité du fait de leurs caractéristiques intrinsèques : le conducteur n'aurait ni le temps ni l'envie de s'engager attentionnellement dans la conversation. Une alternative à ce positionnement serait de considérer que ces conversations de courte durée entraînent, au même titre que des conversations plus longues et plus engageantes, une réorientation de l'attention du conducteur, altérant son contrôle de la scène routière. Le point de focalisation attentionnel du conducteur passant du traitement des informations relatives à sa tâche principale de conduite à celui des informations relatives à sa communication téléphonique, une dégradation du traitement visuel de l'environnement routier devrait être observée au même titre qu'il l'a déjà été montré pour des conversations plus longues et engageantes. Inspiré par l'expérience 3 de Strayer et al. [15], l'étude présente investigue l'influence de communications téléphoniques de très courtes durées et peu engageantes (basées sur un échange de questions/réponses entre le conducteur et l'expérimentateur au téléphone n'appelant pas de discussions complémentaires) sur le comportement attentionnel au volant. Pour ce faire, 17 conducteurs ont conduit sur un parcours simulé autoroutier $2 \times 2$ voies, alternant des sections de conduite seule et de conduite en téléphonant. Trente panneaux sur portique étaient éparpillés de façon pseudo-aléatoire sur le parcours, de telle sorte que la moitié soit positionnée sur les sections de conduite seule et l'autre moitié sur les sections de conduite en téléphonant. Soutenant l'hypothèse que les conversations téléphoniques même de durée très limitée ( $>$ à 20 secondes) entraînent une réorientation attentionnelle $\mathrm{du}$ conducteur du traitement de la scène routière à celui de l'échange téléphonique, nous nous attendons à observer un phénomène d'aveuglement inattentionnel, marqué par une dégradation des performances de reconnaissances des informations neutres (noms communs) présentées sur les panneaux sur portique sans altération de la durée et de la fréquence des fixations oculaires sur ces derniers. Les informations présentées sur les panneaux sont des informations non relatives à l'activité immédiate du conducteur, permettant de mesurer directement (par le biais de l'analyse des mouvements oculaires) et indirecte (par l'analyse $\mathrm{du}$ nombre de mots présentés correctement reconnus dans les deux situations de conduite) son comportement visuo-attentionel.

\section{Méthode}

\subsection{Participants}

17 conducteurs ( 8 femmes et 9 hommes) âgés en moyenne de 29,4 ans (écart-type 4,2 ans) ont participé à cette étude. Tous conduisaient au moins $10000 \mathrm{~km} / \mathrm{an}$ et avaient leur permis de conduire depuis au moins 5 ans. Un examen médical préalable a révélé que tous les participants étaient en bonne santé, et avaient à la fois une bonne vue (score supérieur à $10 / 10^{\mathrm{e}}$ sur l'échelle de Monoyer) et une bonne audition (rapporté par les participants lors de l'interrogatoire médical). Le protocole de l'étude a été examiné et appliqué selon la procédure de la loi sur les recherches biomédicales chez le sujet volontaire sain (Loi Huriet-Sérusclat). L'université Fédérale de Toulouse ne bénéficiant pas d'un comité d'éthique au moment de la conception de ce protocole, il n'a pu lui être soumis.

\subsection{Matériel et méthode}

Le simulateur de conduite : il s'agit d'un simulateur de conduite statique Siemens VDO, couplé à un système de poursuite oculaire (ASL) et à un système audio. Le simulateur de conduite statique est constitué d'un véhicule (Renault 19) associé à une unité de visualisation interactive. L'écran est placé à une distance de $3,5 \mathrm{~m}$ du conducteur. La taille de l'écran est de 4 mètres de large et de 3 mètres de haut, impliquant un champ de vision de $180^{\circ}$. Les caractéristiques du circuit correspondent à celles d'une autoroute à $2 \times 2$ voies sans aucun autre véhicule en circulation. Le parcours expérimental simulé faisait 55 kilomètres, jalonné par 30 panneaux autoroutiers présentés à intervalle varié (en moyenne, tous les $1,5 \mathrm{~km}+/-800 \mathrm{~m}$ ). Les panneaux autoroutiers sont présentés sur portique, leur taille dans l'environnement simulé est de $2,5 \mathrm{~m}$ de haut et de $10 \mathrm{~m}$ de large (les panneaux sont présentés à $5,5 \mathrm{~m}$ de la route simulée, Voir figure 1). Au centre de ces panneaux des noms communs issus de 15 catégories (telles que : " équipement de la voiture », " fleur », « fruit») de noms communs concrets (tels que : «volant», «tulipe», « pomme») étaient écrits. La moitié apparaissait en condition de conduite seule et l'autre en condition de conduite avec conversation courte. Les conducteurs ne recevaient aucune consigne relative à ces panneaux. La taille des caractères sur les panneaux était de 1,3 $\mathrm{m}$. Les panneaux et les mots écrits dessus étant des objets intégrés à une scène routière, leurs métrages respectifs sont relatifs à la scène simulée et non à l'espace physique réel.

Le système d'enregistrement des mouvements oculaires : l'enregistrement des mouvements oculaires a été effectué en utilisant un oculomètre ASL501 de la société 


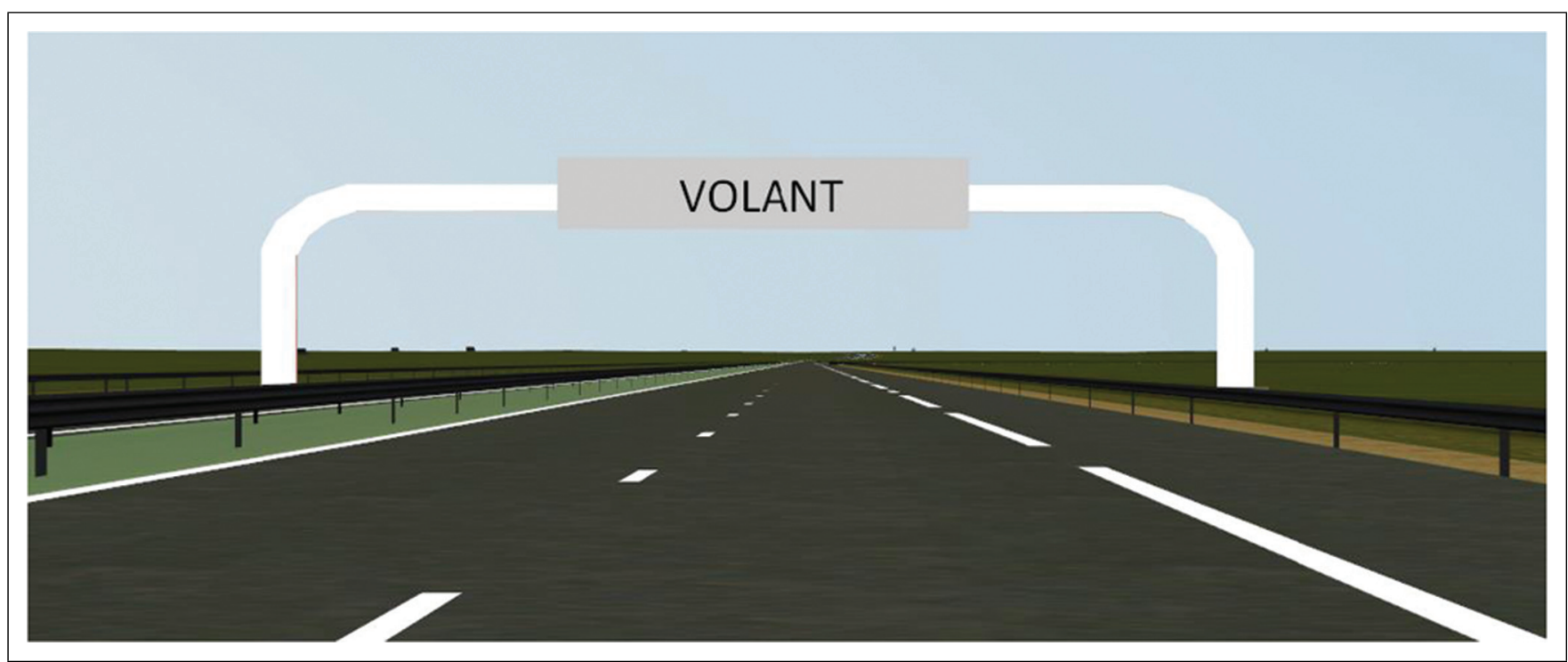

Fig 1 Panneau autoroutier sur portique présentant un nom commun

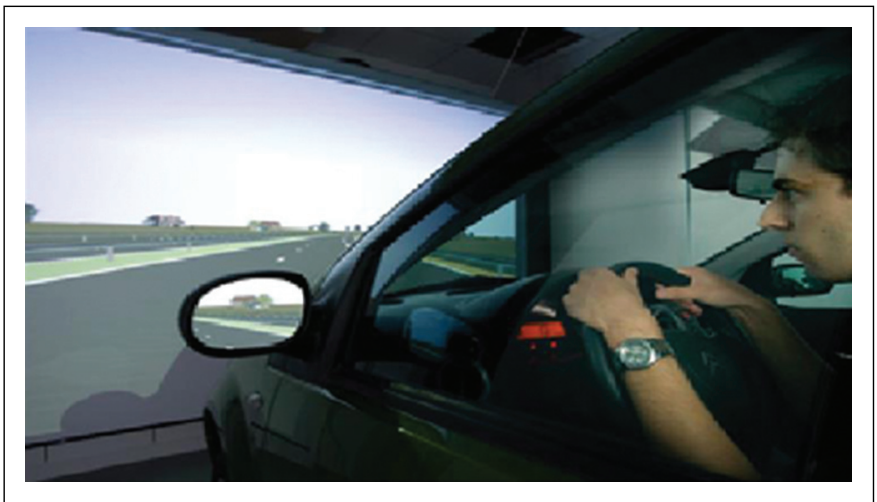

Fig 2 Illustration du dispositif de simulation

Applied Science Laboratories. Cet oculomètre possède une fréquence d'échantillonnage de $50 \mathrm{~Hz}$ et une résolution spatiale de $1^{\circ}$ (spécifications techniques fournies par le fabricant). L'ensemble des données oculométriques a été extrait en utilisant les algorithmes fournis par la société ASL. La distance oculomètre-œil était de $60 \mathrm{~cm}$.

Le système audio : Le système audio est constitué de 2 haut-parleurs intégrés à l'habitacle et d'un microphonecasque sans fil (placé sur la tête du conducteur) reliés à un ordinateur localisé dans une salle de contrôle permettant respectivement de s'adresser au conducteur vocalement et d'entendre ses réponses.

Les échanges téléphoniques : l'objet de cette étude étant d'évaluer l'impact de conversations courtes et informatives (plutôt qu'à caractère émotionnel ou attractif) sur l'attention du conducteur, nous avons sélectionné deux types d'épreuves permettant plusieurs échanges courts entre le conducteur et son interlocuteur : une épreuve de poursuite de mot (impliquant un traitement verbal) et une épreuve d'imagerie spatiale (impliquant un traitement visuo-spatial). L'épreuve verbale [19] consistait en la génération de mots nouveaux commençant par la dernière lettre d'un mot énoncé par l'interlocuteur téléphonique (Par exemple, énoncer un mot nouveau commençant par la dernière lettre $\mathrm{du}$ mot « coucou », une réponse possible est « usine »). L'épreuve d'imagerie spatiale [20], consistait à identifier la forme d'une lettre (fermée ou ouverte) de l'alphabet (considérée écrite en majuscule). Par exemple, visualiser la lettre « $\mathrm{E} »$ ou « $\mathrm{O}$ » en majuscule et dire « ouverte » ou « fermée » (La réponse attendue ici était «ouvert» pour « $\mathrm{E} »$, et fermée pour « $\mathrm{O} »)$. La durée de chaque conversation téléphonique était d'environ 20 secondes, correspondant à 4 échanges entre le conducteur et son interlocuteur téléphonique. Chacune des deux épreuves était répétée 12 fois sur le parcours.

Le test de reconnaissance incident des panneaux routiers : ce test informatisé de reconnaissance comportait 45 noms communs, dont les 30 présentés sur les panneaux du parcours simulé et 15 nouveaux appartenant aux mêmes catégories que les mots présentés durant le parcours. Les participants devaient indiquer pour chacun s'il était ancien (présenté sur le parcours) ou nouveau (non présenté sur le parcours). Ce test se déroulait dans une salle attenante à celle du simulateur de conduite, juste après la tâche de conduite simulée.

\subsection{Procédure}

L'étude se déroulait en trois temps : 1. la familiarisation à la conduite simulée seule et aux épreuves téléphoniques. Elle 
permettait à l'expérimentateur de présenter les consignes relatives à la conduite sur circuit simulé, et à s'assurer de leur bonne compréhension et de leur respect. Les conducteurs recevaient comme consigne de rouler entre 110 et $130 \mathrm{~km} / \mathrm{h}$ sur la voie de circulation droite, et de maintenir leur position sur cette voie. 2. la phase expérimentale consistait à conduire sur une portion d'autoroute simulée, et à répondre à l'interlocuteur téléphonique lorsque celui-ci posait des questions. Les phases de conduite seule et de conduite en situation de conversation téléphonique étaient organisées dans un ordre pseudo-aléatoire. Aucune information n'était donnée aux conducteurs concernant les panneaux autoroutiers jalonnant le circuit. Au total, le parcours routier était réalisé en 37 minutes environ, intégrant au total 8 minutes de conduite avec conversation intégrant un panneau et 8 minutes de conduite seule intégrant un panneau. Les analyses réalisées sur les paramètres de conduite et les paramètres oculomoteurs ont été menées sur les deux périodes de conduite exclusivement, de telle sorte que les données comparées soient effectivement comparables 3 . À l'issue de la conduite, un test de reconnaissance incidente des panneaux routiers était proposé aux conducteurs.

Trois types de données ont été analysés : 1 . les paramètres véhicules de positionnement longitudinal : la vitesse moyenne, et de positionnement latéral du véhicule : le pourcentage de débordement imminent $(<0,6$ secondes) sur la bande d'arrêt d'urgence. Cette mesure du positionnement latéral est couramment appelée Time-to-Lane-Crossing (TLC), que l'on pourrait traduire par la latence séparant le véhicule du débordement de la ligne blanche. Développée dans le cadre d'études sur l'endormissement au volant, on considère que dans un contexte de conduite autoroutière lorsque cette latence est inférieure à $0,6 \mathrm{sec}$., un risque fort de sortie de route imminente est présent. 2. les paramètres oculomoteurs correspondant aux fixations oculaires sur les panneaux (taille de la pupille, fréquence et durée des fixations oculaires). 3. Enfin, la performance au test de reconnaissance incidente en termes de pourcentage de reconnaissance correcte et de temps moyen de reconnaissance. L'analyse préliminaire des résultats n'ayant montré aucun effet simple $(F>1)$ ou d'interaction $(F>1)$ de la variable indépendante type de conversation (épreuve verbale vs. épreuve visuo-spatiale), nous avons fait le choix d'écarter cette variable de l'analyse. Aussi, celle-ci ne portera que sur l'étude de l'impact de la condition de conduite (avec ou sans conversation) sur les trois ensembles de mesure précédemment présentés.

\section{Résultats et discussion}

Trois participants ( 1 femme et 2 hommes) ont été écartés de l'analyse, du fait de problèmes techniques lors de l'enregistrement des données oculométriques. L'analyse porte donc sur 14 participants au total.

\subsection{Mesures on-line de l'impact de conversations téléphoniques courtes sur le comportement du conducteur sur le parcours simule}

Une analyse de variance (ANOVA) à mesures répétées prenant comme facteur intra-sujet la condition de conduite (seule vs. avec conversation) a été menée respectivement sur les deux groupes d'indicateurs du comportement de conduite suivant : les paramètres véhicules, intégrant : la vitesse moyenne, le pourcentage de TLC $<0,6$ secondes, et les paramètres oculométriques, incluant le diamètre pupillaire, la fréquence et la durée des fixations oculaires. En accord avec Haigney, Taylor et Westerman [20], nous nous attendons tout d'abord à une diminution de la vitesse moyenne de conduite en situation de conduite en téléphonant, signe de la mise en œuvre d'un processus compensation du risque. Par ailleurs, suivant les résultats de Strayer et ses collaborateurs [15], une augmentation de la dilatation pupillaire en condition de conduite en téléphonant est attendue, signe d'une augmentation de la charge de travail mental du conducteur.

Les paramètres véhicules : l'ANOVA à mesures répétées portant sur les paramètres véhicules ne met en évidence aucun effet de la condition de conduite (Vitesse : $\mathrm{F}(1,13)=$ 1,507 ; NS ; TLC $<0,6$ sec. ; F $<1$ ). Les participants roulent en moyenne à $93 \mathrm{~km} / \mathrm{h}$ et ont un pourcentage de TLC moyen de $19 \%$. L'absence d'effet lié à la condition de conduite sur les paramètres véhicules peut trouver une explication dans la valeur même de ces paramètres : alors que les instructions les invitaient à rouler entre 110 et $130 \mathrm{~km} / \mathrm{h}$, les conducteurs ont finalement adopté une vitesse de conduite beaucoup plus faible, leur permettant de répondre correctement aux sollicitations de l'expérimentateur sans modifier leur comportement de conduite courant sur le circuit. Les paramètres oculométriques : L'ANOVA à mesures répétées portant sur le diamètre pupillaire montre une augmentation de la taille de la pupille des conducteurs entre la condition de conduite seule $(0,50 \mathrm{~mm})$ et la condition de conduite avec conversation $(0,54 \mathrm{~mm} ; \mathrm{F}(1,13)=32,798$; $\mathrm{MSe}=116,88 ; \mathrm{p}<0,001)$. Ce résultat est en accord avec les travaux antérieurs montrant une augmentation de la dilatation pupillaire relative à la charge mentale de travail du conducteur [20]. Enfin, l'ANOVA à mesures répétées conduite respectivement sur la fréquence et la durée des fixations oculaires lors des fixations oculaires sur les panneaux ne révèle aucun effet de la condition de conduite sur ces deux variables (respectivement, sur la fréquence des fixations oculaires : $\mathrm{F}<1$; Sur la durée des fixations oculaires n'est pas significatif : $\mathrm{F}<1$ ). 


\subsection{Mesures off-line de l'impact de conversations téléphoniques courtes sur le comportement du conducteur sur le parcours simule}

Une dernière ANOVA à mesures répétées a été conduite sur les mesures de la performance au test de reconnaissance incidente (le pourcentage de reconnaissance correcte et les temps moyens de réponse). Un effet principal de la condition de conduite sur le pourcentage de reconnaissance correcte est observé $(\mathrm{F}(1,13)=9,802 ; \mathrm{MSe}=3192,89 ; \mathrm{p}<0,01)$, passant de $67,6 \%$ de reconnaissance correcte en condition de conduite seule à $46.3 \%$ en conduite avec tâche ajoutée. Enfin, une dégradation significative des temps de réaction moyens de reconnaissance est observée de la condition de conduite seule $(1046 \mathrm{~ms})$ à la condition de conduite avec tâche $(1272 \mathrm{~ms})(\mathrm{F}(1,13)=5,318 ; \mathrm{MSe}=281$ 144,045 ; $\mathrm{p}<0,05)$. Ces résultats, couplés aux précédents portant sur les fixations oculaires des panneaux, vont dans le sens d'une inattention du conducteur à la scène routière lors de communications téléphoniques de très courte durée. Ainsi, même dans le cadre très circonscrit de communications n'excédant pas quatre échanges, le conducteur regarde les objets qui l'environnent sans les voir, sans les traiter attentionnellement. Il s'avère ici important de rappeler que les mots présentés sur les panneaux sur portique étaient des noms communs, n'ayant aucune pertinence immédiate pour le conducteur dans son activité principale. Les panneaux publicitaires utilisés par Strayer et ses collaborateurs [15] revêtaient ces mêmes caractéristiques. En cela, nos résultats étendent ceux menés par Strayer sur le comportement visuo-attentionnel au volant. Des études complémentaires permettraient de vérifier si ce même phénomène d'aveuglement inattentionnel serait observé pour des objets de la route ayant une pertinence immédiate avec l'activité de conduite (signaux indicatifs, véhicules, motocyclistes, piétons) lors de conversations de très courte durée et factuelles.

\section{Conclusion}

Téléphoner au volant sur une très courte durée, entraîne une altération du contrôle de l'environnement routier, marqué par un aveuglement inattentionnel aux objets saillants (panneaux présentant un nom commun sur portique) au même titre que des conversations plus longues [15]. Cet effet perturbateur des conversations téléphoniques serait la conséquence d'une réorientation de l'attention du conducteur de la conduite vers le sujet de la conversation téléphonique [17). Même lorsqu'il regarde directement les panneaux, le conducteur est alors moins à même de les traiter du fait que son attention est dirigée ailleurs. L'attention du conducteur étant perturbée, l'encodage des objets saillants de la scène de conduite serait alors altéré. Notre étude conforte cette hypothèse et ajoute à ces conclusions le fait que ce phénomène est observé même dans le cadre de conversations de très courte durée.

Le fait que les mots présentés sur les panneaux routiers étaient, pour la plupart, des noms communs non pertinents dans le contexte de la conduite peut être considéré comme une limite à notre étude. Les conducteurs auraient alors pu minimiser le traitement de ces panneaux pour se concentrer sur leur activité principale. Néanmoins, notre étude portant sur la comparaison du comportement des mêmes conducteurs en situation de simple vs double tache permet de considérer l'effet relatif des conversations de courte durée sur le comportement attentionnel au volant. Il est à souligner que l'utilisation de données physiologiques fut essentielle dans l'identification d'un phénomène d'aveuglement inattentionnel chez le conducteur alors qu'il téléphone. L'intégration de telles mesures en complément des données comportementales plus classiques s'avère ici d'une grande pertinence s'agissant de l'étude du comportement de conduite.

Si l'activité la plus couramment étudiée au volant est celle de converser avec un tiers (qu'il s'agisse de conversations portant sur un thème particulier, ou sur la réalisation d'une épreuve cognitive), ce dernier ne passe finalement qu'une faible part de son activité de conduite à téléphoner (estimée à $6,7 \%$ du temps de trajet journalier, [20]). A contrario, d'autres activités peu étudiées à ce jour, sont à la fois beaucoup plus fréquentes et sources certaines d'inattention. Il s'agit du traitement par le conducteur de ses pensées internes $[19,1]$. Proche du concept de « divagation de l'esprit » (mind-wandering ; développé par Antrobus, [21]), elles regroupent l'ensemble des pensées non liées à l'activité de conduite, survenant dans un contexte de conduite peu coûteux en ressources attentionnelles. Parmi elles, on distingue les pensées ruminatives (d'ordre émotionnel) des pensées distractives (d'ordre factuel), les pensées relatives à un souvenir passé (récupération rétrospective) des pensées relatives à un événement à venir (souvenir prospectif) ou à la résolution d'un problème particulier. L'étude des pensées ou divagations de l'esprit reste encore limitée du fait des difficultés à les opérationnaliser expérimentalement, et en mesurer les effets. Pourtant, leur investigation est déterminante afin d'en parer les effets tant comportementaux que physiologiques au volant. Rappelons ainsi qu'une étude menée en 2004 par Lagarde et al. [22], a mis en évidence que les conducteurs subissant un événement dramatique (comme la perte d'un proche ou un divorce), montraient un risque quatre fois plus important d'accident de la route. Le conducteur, préoccupé par ses pensées internes serait moins attentif à son activité de conduite. Cette hypothèse est soutenue par des travaux récents montrant que l'état de tristesse et le traitement de 
pensées ruminatives entraînent une altération spécifique de la fonction d'orientation de l'attention, liée à un traitement plus superficiel des indices spatiaux [6,8]. Récemment, He, Becic, Lee et McCarley [23] ont aussi montré dans une étude empirique sur simulateur de conduite, que les divagations de l'esprit entraînent une dégradation du contrôle attentionnel, marqué par un balayage visuel focalisé sur la zone proximale avant du véhicule. Cette altération de la projection du regard vers le lointain aurait pour conséquence une moins bonne prise en considération des événements à venir sur la route, réduisant les capacités d'anticipation des conducteurs.

Si la conduite automobile a été longtemps considérée comme une tâche coûteuse en ressources de traitement, à forte charge mentale de travail pour le sujet humain, elle est désormais vue comme une tâche contrôlée attentionnellement, impliquant des processus antagonistes de sélection des informations pertinentes/inhibition des informations non pertinentes. L'enjeu de ces prochaines années sera donc l'étude des variations du contrôle attentionnel du conducteur au volant, afin de mieux en appréhender les manifestations tant comportementales que physiologiques. C'est sur la base de ces recherches que naîtront à terme, de nouveaux systèmes embarqués permettant de parer les périodes d'inattention du conducteur au volant. C'est également sur la base de ces recherches qu'un certain nombre de préconisations au conducteur peuvent déjà être avancées dans une démarche d'information et d'éducation à la conduite. Dans cette perspective, notre étude souligne le risque inhérent à utiliser son téléphone mobile (kit main libre) en conduite, même dans le cadre de conversations de très courtes durées, et portant sur des informations peu attractives. Si l'on pouvait, jusqu'à présent, minimiser les risques relatifs au fait de répondre à des appels de très courte durée, comme l'appel d'un collègue s'interrogeant sur le lieu de la réunion à venir, ou à celui de son conjoint pris dans les embouteillages alors qu'il devait aller chercher les enfants, cette étude montre que leur impact sur la qualité de la prise d'information sur la scène routière est du même ordre qu'une conversation à la fois plus longue et plus attractive.

\section{Bibliographie}

1. Lemercier, C., Cellier, J-M (2008) Les défauts de l'attention en conduite automobile: inattention, distraction, et interférence. Le Travail Humain 71(3) : 271-296.

2. Treat, J, Tumbas, N, McDonald, S, Shinar, D, Hume, R, Mayer, $\mathrm{R}$ et al., (1979). Tri-level study of the causes of traffic accidents: Executive summary. Technical Report DOT HS-805 099, Institute for Research in Public Safety, Bloomington : University of Indiana.

3. Van Elslande, P, Perez, E, Nachtergaele, C (2005). De la vigilance à l'attention: Déclinaison des problèmes liés à l'état psychophysiologique et cognitif du conducteur, et analyse de leur influence sur les mécanismes d'accidents. Technical Report VIGA, Institut National de Recherche sur les Transports et leur Sécurité.

4. Hendricks, DL, Fell, JC, Freedman, M (1999). The relative frequency of unsafe driving acts in serious traffic crashes (Report DTNH22-94-C-05020). Washington, DC: National Highway Traffic Safety Administration.

5. Rumar, K (1990). The basic driver error : Late detection. Ergonomics $33: 1281-1290$.

6. Regan, MA, Hallett, C, Gordon, CP (2011) Driver distraction and driver inattention: Definition, relationship and taxonomy. Accident Analysis and Prevention 43:1771-1781.

7. Pêcher, C, Lemercier, C (2011) Sadness and the orienting of attention. 17th Meeting of the European Society for COgnitive Psychology, 29th September- 2d October, San Sebastian, Spain.

8. Fan, J, McCandliss, D, Sommer, T, Raz, A, Posner, MI (2002) Testing the efficiency and the independance of attentional networks. Journal of Cognitive Neurosciences 19(1\&2) : 340-347.

9. Pêcher, C, Quaireau, C, Lemercier, C, Cellier, J-M (2011) The effects of inattention on selective attention: How sadness and ruminations alter attention functions tested with the Attention Network Test. European Review of Applied Psychology 61 : 43-50.

10. Mack, A, Rock, I (1998) Inattentional Blindness, MIT Press.

11. Hills, BL (1980)Vision, visibility, and perception in driving. Perception 9(2): 183-216.

12. Most, SB, Simons, DJ, Scholl, BJ, Jimenez, R, Clifford, E, Chabris, CF (2001) How not to be seen: The contributions of similarity and selective ignoring to sustained inattentional blindness. Psychological Science 12: 9-17.

13. Yantis, S (1993) Stimulus-driven attention capture. Current Directions in Psychological Science $2: 156-161$.

14. Langham, M, Hole, G, Edwards, J, O’Neil, C (2002) An analysis of 'looked but failed to see' accidents involving parked police vehicles. Ergonomics 45(3) : 167-185.

15. Most, SB, Astur, RS (2007) Feature-based attentional set as a cause of traffic accidents. Visual Cognition 15(2) : 125-132.

16. Strayer, DL, Drews, FA, Johnston, W (2003) Cell phone-induced failures of visual attention during simulated driving. Journal of Experimental Psychology : Applied 9(1) : 23-32.

17. Strayer, DL, Cooper, JM, Drews, FA (2004) What do drivers fail to see when conversing on a cell phone? In the Proceedings of the 48nd Annual Meeting of the Human Factors and Ergonomics Society, 2213-2217.

18. Brusque, C, Alauzet, A (2008) Analysis of the individual factors affecting mobile phone use while driving in France: Sociodemographic characteristics, car and phone use in professional and private contexts. Accident Analysis \& Prevention 40(1) : 35-44.

19. Gugerty, L, Rakauskas, M, Brooks, J (2004) Effects of remote and in-person verbal interactions on verbalization rates and attention to dynamic spatial scenes. Accident Analysis and Prevention 36: 1029-1043

20. Recarte, MA, Nunes, LM (2003) Mental workload while driving: effects on visual search, discrimination, and decision making. Journal of Experimental Psychology: Applied 9:119-137.

21. Haigney, D, Taylor, R, Westerman, S (2000) Concurrent mobile (cellular) phone use and driving performance: task demand characteristics and compensatory processes. Transportation research Part F 3: 113-121.

22. Antrobus, JS (1968) Information theory and stimulus-independent thought. British Journal of Psychology 59: 423-430.

23. Lagarde, E., Chastang, J-F, Gueguen, A, Coeuret-Pellicer, M, Chiron, M, Lafont, S (2004) Emotional stress and traffic accidents: the impact of separation and divorce. Epidemiology 15: 762-766.

24. He, J, Becic, E, Lee, Y-C, McCarley, JS (2011) Mind wandering behind the wheel: Performance and oculomotor correlates. Human Factors 53(1): 13-21. 
